\title{
Vectorlike leptons as the tip of the dark matter iceberg
}

\author{
James Halverson \\ Kavli Institute for Theoretical Physics, University of California \\ Santa Barbara, CA 93106-4030 USA \\ Nicholas Orlofsky and Aaron Pierce \\ Michigan Center for Theoretical Physics (MCTP), \\ Department of Physics, University of Michigan \\ Ann Arbor, MI 48109-1040 USA
}

(Dated: February 28, 2022)

\begin{abstract}
A vectorlike lepton could make up a tiny fraction of the dark matter. Its large $Z$-boson mediated direct detection cross section can compensate for the small relic abundance, giving rise to an interesting signal at future experiments - perhaps even the first one detected. We discuss how such a scenario might arise in the context of a simple non-thermal cosmology and investigate bounds from direct detection experiments and whether this scenario might be probed at the LHC. Searches for disappearing tracks appear promising.
\end{abstract}

\section{INTRODUCTION}

A well-motivated and minimal possibility is that dark matter interactions in direct detection experiments are mediated by force carriers of the Standard Model: the $Z$-boson or the Higgs boson. The $Z$-boson gives a spinindependent $(\mathrm{SI})$ dark matter-nucleon scattering cross section $\sigma_{\mathrm{SI}} \approx 10^{-38} \mathrm{~cm}^{2}$ for masses $\sim 100 \mathrm{GeV}$. With bounds at this mass approaching $10^{-45} \mathrm{~cm}^{2}[1,2]$, it at first seems counterproductive to consider dark matter with full strength couplings to the $Z$. Indeed, the canonical approach is to forbid the $Z$-mediated operator relevant for SI scattering $\mathcal{O}_{Z}=\left(\bar{q} \gamma_{\mu} q\right)\left(\bar{X} \gamma^{\mu} X\right)$, e.g., by making the dark matter Majorana. (In this case, Higgs boson exchange may yield direct detection cross sections close to the typical bounds, see e.g. [3, 4].)

However, it is only necessary to forbid this operator if the relic makes up the entirety of the dark matter. A relic may comprise a miniscule fraction of the dark matter, but its enormous direct detection scattering cross section can lead to an interesting signal (See [5] for related work on detecting a subdominant component of the DM in the context of the MSSM.). We will see dilution by the necessary amount is possible in simple cosmologies. We briefly discuss ways in which such a particle - which could be the first discovery at direct detection experiments - might be disentangled from the dominant dark matter using information from both colliders and direct detection.
As a concrete example, consider the addition of a vectorlike pair of doublets $X, \bar{X}$ to the minimal supersymmetric standard model (MSSM). What mass scale might we expect for these doublets? Some mechanism must generate a mass $\mu$ for the Higgsinos of the MSSM. Whether this is the Giudice-Masiero mechanism [6], the vacuum expectation value of a singlet (as in the next-to minimal supersymmetric standard model [7]), or a Dbrane instanton [8], it is plausible that whatever generates $\mu$ would also generate a mass $\mu_{X}$ at the same scale. Naturalness arguments then indicate a $\mu \sim \mu_{X} \sim 100$ $\mathrm{GeV}-1 \mathrm{TeV}$. If $X, \bar{X}$ have an unbroken $X \rightarrow-X$ symmetry, the Dirac fermion will comprise a component of the dark matter. Because it is Dirac, it has full-strength direct detection cross section per nucleon, e.g. $[9,10]$ :

$$
\sigma \approx \frac{G_{F}^{2}}{2 \pi} \mu_{X N}^{2} \frac{1}{A^{2}}\left[\left(1-4 \sin ^{2} \theta_{W}\right) Z-(A-Z)\right]^{2},
$$

where $\mu_{X N}$ is the dark matter-nucleon reduced mass.

Another potential motivation for novel vectorlike doublets arises via string theory. There Ref. [11] has shown that there exist additional constraints on the chiral spectrum of $\mathrm{SU}(2)$ gauge theories which ensure anomaly cancellation in nucleated D-brane theories. These constraints go beyond standard anomaly cancellation in the $\mathrm{SU}(2)$ theories and can require the existence of electroweak exotics; see [12] for particle physics implications. In weakly coupled orientifold compactifications, doublet quantum numbers for the exotics are a likely possibility. 
The exotic states arise from open strings, which selects out $\mathrm{SU}(2)$ singlets, doublets, and triplets as the only possibilities. If one further requires that one end of the open string ends on a D-brane corresponding to a novel symmetry (perhaps related to the stability of the $X$ ), then doublet quantum numbers are uniquely selected. A string scale mass term for the doublets is forbidden by symmetry, rendering their presence at the $\mathrm{TeV}$ scale even more plausible. If the low energy theory is the Standard Model plus these exotics, gauge couplings approximately unify at $10^{14} \mathrm{GeV}[13,14]$.

We will work under the assumption that a new Dirac $\mathrm{SU}(2)$ doublet $X$ exists in the $100 \mathrm{GeV}$ to $1 \mathrm{TeV}$ range. We address implications for direct detection experiments and sketch how the dilution necessary to bring it into compliance with experimental bounds might be accomplished in a simple non-thermal cosmology. Finally, we discuss prospects for probing such a doublet at the LHC.

\section{DIRECT DETECTION AND COSMOLOGY}

We have used MicrOMEGAs [15] to calculate the spinindependent cross section of $X$ and verified that it is consistent with Eq. (1). We also used it to calculate the $X$ relic abundance assuming a standard thermal freezeout. It is well approximated by $\Omega_{X} h^{2} \simeq 0.1\left(\frac{\mu}{1 \mathrm{TeV}}\right)^{2}[16]$. An $X$ produced with a standard thermal history is well excluded by current direct detection bounds. To evade current bounds from LUX [1], $X$ with $\mu_{X}=100 \mathrm{GeV}(1$ $\mathrm{TeV})$ must have a tiny relic density $\Omega_{X} / \Omega_{\text {cdm }} \lesssim 5 \times 10^{-7}$ $\left(4 \times 10^{-6}\right)$, where $\Omega_{\mathrm{cdm}} h^{2}=0.1199 \pm 0.0027$ [17]

One possibility is to simply declare a smaller relic abundance by fiat. Indeed, we could imagine that there is thermal freeze-out with a subsequent dilution by e.g. late time inflation. Interestingly, however, the maximum dilution is limited if the baryon number is generated before this dilution. Even if the baryon asymmetry proceeds by an extraordinarily efficient mechanism like AffleckDine (for a review see [18]), where the baryon to photon ratio could be as large as $\mathcal{O}(1)$, consistency with the current ratio imposes a maximum dilution factor of $10^{9}[19]$. Then, the dark matter densities would range from $\Omega_{\mathrm{dil}} h^{2} \approx 10^{-12}-10^{-10}$ for $\mu_{X}=100-1000$ $\mathrm{GeV}$. But given the large direct detection cross sections, it should be possible to probe relic abundances of $\Omega_{\text {dil }} h^{2} \approx 5 \times 10^{-11}-10^{-11}$ without running afoul of the neutrino background [20]. A 1 ton Xe experiment might be sensitive to relic densities perhaps a hundred times these; therefore, it is possible to almost completely probe this scenario of arbitrary dilution. We explore a perhaps better motivated possibility below, where we discuss a more concrete cosmology. In that case, the relic abundance is expected be less diluted, and therefore the likelihood of direct detection is even greater.

This model gives a characteristic material dependence at direct detection experiments. The ratio of measured cross section per nucleon at experiments composed of Xenon, Germanium, and Argon would be $1: 0.89: 0.86$. Observing the deviation of these ratios from unity will be challenging but would be powerful evidence for this scenario. Also, if the mass is close to $100 \mathrm{GeV}$, it is possible to make a determination of the $X$ mass via an examination of the recoil spectrum, e.g., [21]. This mass could then be correlated with collider discoveries, see below.

\section{A. Non-thermal production via modulus decay}

The late decay of a scalar field $\phi$ can modify the dark matter relic abundance. This occurs if the energy density of the universe becomes $\phi$-dominated until the time of $\phi$ decay, which can then both produce dark matter and provide substantial entropy generation as it reheats the universe to a temperature $T_{\mathrm{RH}}$. Such cosmologies are well-motivated in string compactifications, which typically contain many light scalar fields in the form of stabilized moduli. Another possibile motivation is supersymmetric axion models - the saxion could play the role of $\phi$ and the axion and lightest neutralino or axino could make up (some or all of) the remaining dark matter [22].

$T_{\mathrm{RH}}$ is model dependent, but it is bounded by phenomenological requirements. First, to ensure that the successful predictions of big bang nucleosynthesis (BBN) are not spoiled, $T_{\mathrm{RH}} \gtrsim T_{\mathrm{BBN}} \simeq 5 \mathrm{MeV}$ [23]. Second, to accommodate an alternative production mechanism, 
it must be below the thermal freeze-out temperature, $T_{\mathrm{RH}} \lesssim T_{\mathrm{fo}} \simeq \mu_{X} / 20$. We will see that consistency with direct detection bounds will place further limits on $T_{\mathrm{RH}}$.

$T_{\mathrm{RH}}$ is determined by the decay rate $\Gamma_{\phi}=c_{\phi}^{2} m_{\phi}^{3} / M_{P}^{2}$ (perhaps arising from an operator like $\phi G \tilde{G})$. Under the assumption that $\phi$ decay and the subsequent thermalization are instantaneous:

$$
\begin{aligned}
T_{\mathrm{RH}} & =\left[\left(\frac{8}{90} \pi^{3} g_{\star}\right)^{-1 / 2} M_{P} \Gamma_{\phi}\right]^{1 / 2} \\
& \simeq 10 c_{\phi}\left(\frac{m_{\phi}}{100 \mathrm{TeV}}\right)^{3 / 2} \mathrm{MeV}
\end{aligned}
$$

where $c_{\phi}$ is a presumably $\mathcal{O}(1)$ constant computable in specific models.

The $X$ relic abundance $\Omega_{X} h^{2}$ depends on $T_{\mathrm{RH}}$ and $b$, the number of dark matter particles produced per $\phi$ decay. In the Boltzmann equations, only the combination $b / m_{\phi}$ appears [23]; accordingly, we will employ the dimensionless parameter $\eta \equiv b\left(100 \mathrm{TeV} / m_{\phi}\right)$. If the $\phi$ branching ratio to $X$ is very small or zero, the $X$ relic density is set by thermal production and freezeout followed by its dilution via the entropy produced in $\phi$ decays. Since the $X$ interaction cross section is large enough to reach chemical equilibrium prior to freeze-out, the (diluted) thermal relic density is parametrized by $[23,24]$,

$$
\Omega_{X} \simeq \frac{T_{\mathrm{RH}}^{3} T_{\mathrm{fo}}}{\left(T_{\mathrm{fo}}^{\text {new }}\right)^{4}} \Omega_{\mathrm{std}} \simeq\left(\frac{T_{\mathrm{RH}}}{T_{\mathrm{fo}}}\right)^{4} \Omega_{\mathrm{std}}, \quad(\eta \text { tiny }),
$$

where $\Omega_{\text {std }}$ is the $X$ relic abundance assuming a standard thermal history (i.e., $T_{\mathrm{RH}}>T_{\mathrm{fo}}$ ).

On the other hand, $\eta$ may be large enough for nonthermal production to dominate over thermal production. Since direct detection bounds require that the relic abundance be much less than the standard thermal abundance, $\eta$ must nonetheless be small. Thus, non-thermal production will not be compensated by annihilations. In this regime [23],

$$
\frac{\Omega_{X}}{\Omega_{\mathrm{cdm}}} \simeq 2 \times 10^{3} \eta\left(\frac{\mu_{X}}{100 \mathrm{GeV}}\right)\left(\frac{T_{\mathrm{RH}}}{\mathrm{MeV}}\right), \quad(\eta \text { small }) .
$$

Using the sum of expressions (3) and (4) (a good approximation to the numerical solution in this small- $\eta$ regime when $T_{\mathrm{RH}}$ is not too close to $T_{\mathrm{fo}}$ ) and assum$\operatorname{ing} T_{\mathrm{fo}} \simeq \mu_{X} / 25$ (in good agreement with MicrOMEGAs

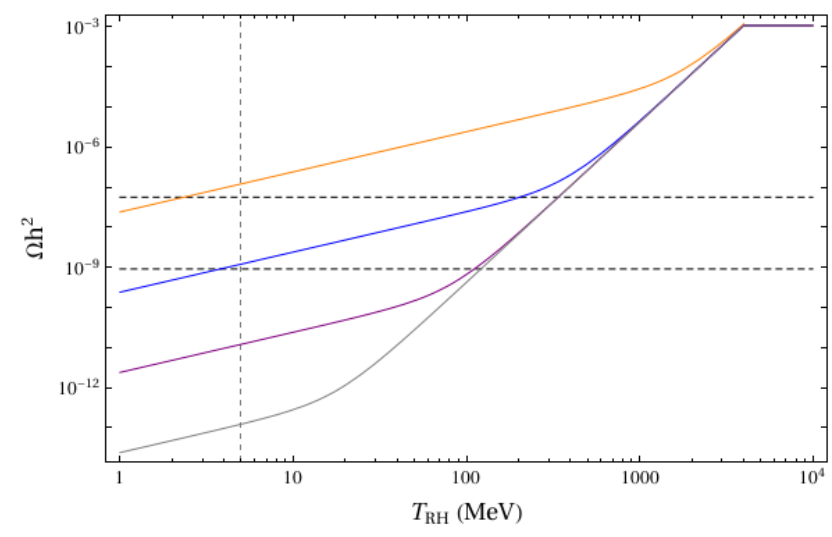

FIG. 1. Relic density as a function of $T_{\mathrm{RH}}$ for various values of $\eta$ for $\mu_{X}=100 \mathrm{GeV}$. The solid lines correspond to, from top to bottom, $\eta=10^{-10}$ (orange), $10^{-12}$ (blue), $10^{-14}$ (purple), and $10^{-16}$ (gray). The horizontal dashed lines correspond to the maximum allowed relic density to evade current LUX (top) and prospective Xe1T (bottom) bounds. The vertical dashed line represents the cutoff of allowable $T_{\mathrm{RH}}$ due to BBN.

and with numerical solutions in [23]), the relic density for $\mu_{X}=100 \mathrm{GeV}$ is plotted as a function of $T_{\mathrm{RH}}$ for various values of $\eta$ in Figure 1, see also Ref. [23]. Also shown are current bounds from LUX [1] and BBN as well as prospective bounds from a ton-scale Xe experiment [25].

Figure 1 indicates that bounds from LUX require $\eta \lesssim$ $10^{-10}$. This is approximately true for any value of $\mu_{X}>$ $100 \mathrm{GeV}$ because the LUX bound on $\sigma_{\mathrm{SI}} \propto \mu_{X}, \Omega_{X} \propto$ $\eta \mu_{X}$ from (4), and the cross section (1) is approximately constant with respect to $\mu_{X}$.

Such small values of $\eta$ require that that the Yukawa coupling which determines $\Gamma_{\phi \rightarrow X X}$ is very small. Is it reasonable to expect such suppression? If $\phi$ is uncharged under the Peccei-Quinn-like symmetry protecting $\mu_{X}$, then the bare operator $\phi X \bar{X}$ is forbidden. However, the effect which generates $\mu_{X}$ will typically also give rise to an effective Yukawa coupling which is $\frac{\mu_{X}}{M_{P}}$ suppressed. For example, if $\mu_{X}$ is generated via singlet expectation value via a coupling $s X \bar{X}$, then the invariant coupling $\frac{1}{M_{P}} \phi s X \bar{X}$ gives an effective Yukawa $\frac{\langle s\rangle}{M_{P}} \phi X \bar{X}$ with $\langle s\rangle \simeq \mu_{X}$. [26] We accordingly parameterize the effective Yukawa coupling as $c_{X} \frac{\mu_{X}}{M_{P}}$, giving,

$$
\Gamma_{\phi X X}=\frac{m_{\phi}}{4 \pi}\left(c_{X} \frac{\mu_{X}}{M_{P}}\right)^{2} \Rightarrow b=\frac{1}{2 \pi}\left(\frac{c_{X}}{c_{\phi}} \frac{\mu_{X}}{m_{\phi}}\right)^{2}
$$

Here, $\Gamma_{\phi X X}$ is the width to both $X^{0} \bar{X}^{0}$ and $X^{+} X^{-}$. 


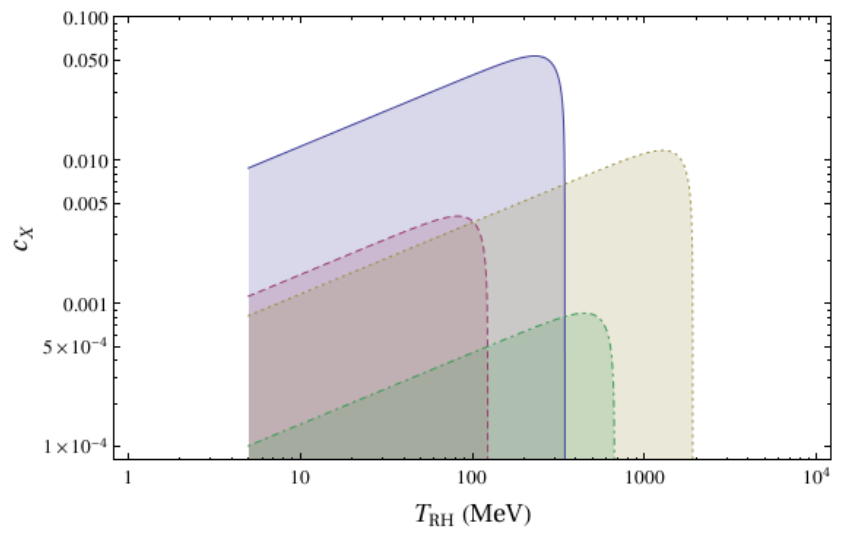

FIG. 2. Allowed regions (shaded) of $c_{X}$ for $\mu_{X}=100 \mathrm{GeV}$ from current LUX (blue solid) and prospective Xe1T (red dashed) bounds and for $\mu_{X}=1 \mathrm{TeV}$ from current LUX (yellow dotted) and prospective Xe1T (green dot-dashed) bounds.

The present constraints on $c_{X}$ are shown in Figure 2. Using Eqs. (4) and (5):

$$
\frac{\Omega_{X}}{\Omega_{\mathrm{cdm}}} \simeq \frac{c_{X}^{2}}{10 \pi}\left(\frac{\mu_{X}}{100 \mathrm{GeV}}\right)^{3}\left(\frac{\mathrm{MeV}}{T_{\mathrm{RH}}}\right) .
$$

This equation determines the upper boundary of the shaded regions. The left boundary is set by BBN: $T_{\mathrm{RH}} \gtrsim T_{\mathrm{BBN}} \simeq 5 \mathrm{MeV}$. The right boundary occurs when Eq. (3) is sufficient to violate (present or future) direct detection bounds.

Absent additional model building, we expect a number of $\mathcal{O}(1)$ contributions to $c_{X}$, so the stringent direct detection bounds already necessitate tuning at the few percent level. The most favored $c_{X}$ region is the one where the relic lies just outside current bounds in Figure 2.

The above assumes a single late-decaying modulus. However, string compactifications often contain $\mathcal{O}(100)$ moduli. For the case of many moduli, the details of the evolution of the dark matter density depend on the initial abundances of the moduli. The single-modulus case is a good approximation to the many-moduli case under the assumption that one modulus $\phi_{i}$ dominates the energy density of the universe before it decays, and that no other modulus comes to dominate the energy density any time after $\phi_{i}$ decays. Otherwise, several moduli may contribute to the non-thermal production of dark matter. Additionally, decays of the later moduli might dilute contributions from earlier moduli.

\section{COLLIDER PHENOMENOLOGY}

The strongest current collider bound on the $\mathrm{SU}(2)$ doublet comes from LEP2, which bounds $\mu_{X} \gtrsim 95 \mathrm{GeV}$ [27]. We present the prospects for LHC14 in the monojet and disappearing track channels. [28]

\section{A. Monojet $+\mathbb{E}_{\mathbf{T}}$}

The mass splitting between the $X^{ \pm}$and $X^{0}$ particles for mass $\mu_{X}=100$ (200) $\mathrm{GeV}$ is $\delta m \simeq 256$ (295) MeV. This is a finite, calculable effect due to electroweak symmetry breaking [29], analogous to the charged/neutral pion mass splitting. With these splittings, the $X^{ \pm}$decay promptly into $X^{0}+$ invisibly soft $\pi^{ \pm}, e^{ \pm} \nu$, or $\mu^{ \pm} \nu$. Charged and neutral doublet particles will appear as missing energy in the detector. The most recent results for this channel are from ATLAS [30] and CMS [31].

To estimate signal and background, we use MadGraph5 [32], pass to Pythia [33] for MLM matching $[34,35]$, showering, and hadronization, and use PGS [36] for detector simulation, using an anti- $k_{t}$ jet clustering algorithm with $R=.5$. Simulated parton-level events include one or two jets. We simulate the dominant backgrounds $j(j)+(Z \rightarrow \nu \nu, W \rightarrow l \nu$, or $W \rightarrow \tau \nu)$, with $l=e, \mu$; the signal has $j(j)+\left(X^{0} \bar{X}^{0}, X^{+} X^{0}, X^{-} \bar{X}^{0}\right.$, or $\left.X^{+} X^{-}\right)$. Following [30], we apply the following cuts: $(i)$ $p_{\mathrm{T}}\left(j_{1}\right)>p_{\mathrm{T}}^{\text {cut }}$ and $\left|\eta\left(j_{1}\right)\right|<2,(i i) \mathbb{E}_{\mathrm{T}}>p_{\mathrm{T}}^{\text {cut }}$, (iii) no more than 2 jets with $p_{\mathrm{T}}>30 \mathrm{GeV}$ and $|\eta|<4.5$, (iv) $\Delta \phi\left(j_{2}, \mathbb{E}_{\mathrm{T}}\right)>.5,(v)$ lepton vetoes: $p_{\mathrm{T}}(e)>20 \mathrm{GeV}$ and $|\eta(e)|<2.47, p_{\mathrm{T}}(\mu)>7 \mathrm{GeV}$ and $|\eta(\mu)|<2.5$, or $p_{\mathrm{T}}(\tau)>20 \mathrm{GeV}$ and $|\eta(\tau)|<2.3$, and (vi) veto on b-jets.

The significance can be found using

$$
\chi^{2}=\frac{S^{2}}{S+B+\sigma_{B}^{2}} .
$$

Its square root gives the significance. We parameterize the background uncertainty as $\sigma_{B}=\beta_{\text {tot }} B$, remaining agnostic about where the uncertainties originate [37].

Taking $\mathcal{L}=3000 \mathrm{fb}^{-1}$, we present significances in Table I assuming that $\beta_{\text {tot }}$ can be made to be either .03 or .01 at $p_{\mathrm{T}}^{\text {cut }}=500 \mathrm{GeV}$. For comparison, ATLAS and CMS have background uncertainties of $\beta_{\text {tot }} \simeq .04$ and .03, 


\begin{tabular}{l|ll|lll} 
& \multicolumn{2}{|l|}{ Signal $\left(\mu_{X}\right.$ in GeV) } & \multicolumn{3}{|c}{ Backgrounds } \\
& $\mu_{X}=100$ & $\mu_{X}=150$ & $B_{j \nu \nu}$ & $B_{j l \nu}$ & $B_{j \tau \nu}$ \\
\hline Cross section $(\mathrm{fb})$ & 5.78 & 3.28 & 136 & 28.0 & 29.7 \\
$\chi\left(\beta_{\text {tot }}=.03\right)$ & 1.0 & .6 & & & \\
$\chi\left(\beta_{\text {tot }}=.01\right)$ & 3.0 & 1.7 & & &
\end{tabular}

TABLE I. Cross sections of backgrounds and signal (following cuts $(i)-(v i)$ in the text) and signal significances for the monojet $+\mathbb{E}_{\mathrm{T}}$ channel with $\sqrt{s}=14 \mathrm{TeV}, \mathcal{L}=3000 \mathrm{fb}^{-1}$, and $p_{\mathrm{T}}^{\text {cut }}=500 \mathrm{GeV}$.

respectively, for cuts in present monojet analyses which have a comparable number of background events as our projected $p_{\mathrm{T}}^{\text {cut }}$ and luminosity We note that if such a small uncertainty could be maintained at larger $p_{\mathrm{T}}^{\text {cut }}$, the significance could be modestly increased.

Based on our analysis, we conclude the discovery sensitivity of this channel to a $\mathrm{SU}(2)$ doublet appears weak. Our signal and background cross sections are in rough agreement with [38-41], although our estimated backgrounds tend to be a little smaller and our signals a little larger. Thus, we reach the same general conclusions as [38-40] that $5 \sigma$ detection is unlikely in this channel, while a small mass window may be excluded at $2 \sigma$.

\section{B. Disappearing track}

Because of the mass splitting discussed in the previous subsection, the path length of the $X^{ \pm}$in its own rest frame for mass $\mu_{X}=100(200) \mathrm{GeV}$ is a modest $c \tau=$ $1.93(1.19) \mathrm{cm}$ [29]. While these path lengths are difficult to detect, it is possible that some of the particles in the tail of the lifetime distribution might be observed if the production rate is sufficiently high. Thus, low masses may be accessible to future disappearing track studies that search for $X^{ \pm}$before they decay. The most recent results from the ATLAS experiment can be found in [42].

Following the cuts in [42], to obtain an estimate for the expected signal, we use MadGraph to simulate $p p \rightarrow j+$ $\left(X^{+} X^{0}, X^{-} \bar{X}^{0}\right.$, or $\left.X^{+} X^{-}\right)$at parton level, stipulating that $p_{\mathrm{T}}(j)>90 \mathrm{GeV}$ and $|\eta(j)|<5$. Then, in each event with $p_{\mathrm{T}}\left(j_{1}\right)>p_{\mathrm{T}}^{\text {cut }}$ (to be varied), we select the $X^{ \pm}$with $.1<\left|\eta^{\text {track }}\right|<1.9, p_{\mathrm{T}}^{\text {track }}>500 \mathrm{GeV}$, and $p_{\mathrm{T}}^{\text {track }}<1000$ $\mathrm{GeV}$ at $\sqrt{s}=8 \mathrm{TeV}$ (to match what is done in ATLAS) and $1500 \mathrm{GeV}$ at $\sqrt{s}=14 \mathrm{TeV}$. Next, using the known lifetime, we calculate the probability that each passing $X^{ \pm}$would achieve a transverse length of at least $30 \mathrm{~cm}$ before decay, corresponding to the beginning of the first SCT layer in the ATLAS detector. As alluded to above, $X^{ \pm}$that reach the SCT are either highly boosted and/or are in the tail of the lifetime distribution. We assume the efficiency for detection after these cuts is $100 \%$.

Comparing to current limits at $\sqrt{s}=8 \mathrm{TeV}$, for $\mu_{X}=$ $100 \mathrm{GeV}$ and $p_{\mathrm{T}}^{\text {cut }}=200 \mathrm{GeV}$, we estimate $\sigma_{\mathrm{vis}}=.27 \mathrm{fb}$. This is just below the ATLAS 95\% exclusion of $\sigma_{\text {vis }}<.44$ fb (smaller $p_{\mathrm{T}}^{\text {track }}$ cuts in [42] set weaker bounds).

To make projections for LHC14, we must estimate the background. A reliable estimate is difficult, as the dominant background (see Figure 5 of [42]) is from mismeasured tracks. We parameterize the background at $\sqrt{s}=14 \mathrm{TeV}$ and luminosity $\mathcal{L}$ as

$B=B_{8 \mathrm{TeV}}\left(\frac{\mathcal{L}}{\mathcal{L}_{8 \mathrm{TeV}}}\right)\left(\frac{\sigma_{14 \mathrm{TeV}}}{\sigma_{8 \mathrm{TeV}}}\right)\left(\frac{\epsilon_{p_{\mathrm{T}}\left(j_{1}\right)>p_{\mathrm{T}}^{\text {cut }}}}{\epsilon_{p_{\mathrm{T}}\left(j_{1}\right)>90 \mathrm{GeV}}}\right) P_{\mathrm{mis}}$,

where $\mathcal{L}_{8 \mathrm{TeV}}=20.3 \mathrm{fb}^{-1}$ is the luminosity in [42], $B_{8 \mathrm{TeV}}$ is the estimated background in [42], $\sigma_{14 \mathrm{TeV}} / \sigma_{8 \mathrm{TeV}}$ accounts for the increased cross section of the background as collision energy increases (leaving all cuts constant), $\epsilon_{p_{\mathrm{T}}\left(j_{1}\right)>p_{\mathrm{T}}^{\text {cut }}} / \epsilon_{p_{\mathrm{T}}\left(j_{1}\right)>90 \mathrm{GeV}}$ accounts for a cut intended to reduce background, and $P_{\text {mis }}$ parameterizes the potential that the probability for mismeasured tracks may be greater with increased energy and pile-up.

We obtain $B_{8} \mathrm{TeV}$ by integrating the background in Figure 5 of Ref. [42] from the $p_{\mathrm{T}}^{\text {track }}$ cut up to $1500 \mathrm{GeV}$. We approximate $\sigma_{14 \mathrm{TeV}} / \sigma_{8 \mathrm{TeV}} \approx 3$ based on MadGraph simulations of $p p \rightarrow j \nu \nu$, (the dominant monojet $+\mathbb{E}_{\mathrm{T}}$ background). We estimate $\epsilon_{p_{\mathrm{T}}\left(j_{1}\right)>p_{\mathrm{T}}^{\text {cut }}} / \epsilon_{p_{\mathrm{T}}\left(j_{1}\right)>90 \mathrm{GeV}}$ by applying cuts to our simulation of $p p \rightarrow j \nu \nu$. Finally, we assume either $P_{\mathrm{mis}}=1$ or 10 and choose $p_{\mathrm{T}}\left(j_{1}\right)$ cuts to optimize the significance for each case. We underscore that many assumptions have been made to approximate $B$. Quoted backgrounds and significances are estimates.

We show estimated $p_{\mathrm{T}}^{\text {track }}$ distributions of the background and signal at various masses for $\sqrt{s}=14 \mathrm{TeV}$ applying the cut $p_{\mathrm{T}}\left(j_{1}\right)>300 \mathrm{GeV}$ in Figure 3 . For simplicity, we chose a single range $500 \mathrm{GeV}<p_{\mathrm{T}}^{\text {track }}<1500 \mathrm{GeV}$ for all $\mu_{X}$. We found optimizing this range does not affect 


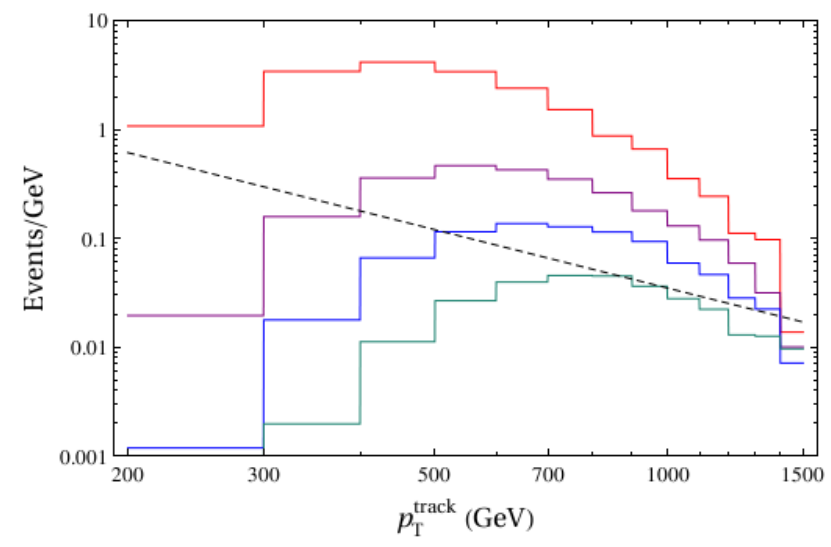

FIG. 3. The $p_{\mathrm{T}}^{\text {track }}$ distribution of the background (black, dashed) and signal (solid) with $p_{\mathrm{T}}\left(j_{1}\right)>300 \mathrm{GeV}$ and $P_{\text {mis }}=1$ at $\sqrt{s}=14 \mathrm{TeV}$ and $\mathcal{L}=3000 \mathrm{fb}^{-1}$. Signal spectra correspond to, from top to bottom, $\mu_{X}=100 \mathrm{GeV}$ (red), 130 $\mathrm{GeV}$ (purple), $150 \mathrm{GeV}$ (blue), and $170 \mathrm{GeV}$ (green).

the significance much.

The estimated backgrounds and signals for various doublet masses at $\sqrt{s}=14 \mathrm{TeV}$ and $\mathcal{L}=3000 \mathrm{fb}^{-1}$ with $500 \mathrm{GeV}<p_{\mathrm{T}}^{\text {track }}<1500 \mathrm{GeV}$ for different $p_{\mathrm{T}}\left(j_{1}\right)$ cuts are shown in Table II.

Significances are estimated using Eq. (7), again parameterizing $\sigma_{B}=\beta_{\text {tot }} B$, and are shown in Figure 4 for luminosities $\mathcal{L}=300$ and $3000 \mathrm{fb}^{-1}$, taking various $P_{\text {mis }}$ and optimizing the $p_{\mathrm{T}}\left(j_{1}\right)$ cut. The most recent ATLAS study has cuts with expected backgrounds of 18-48.5 events and uncertainties of about $25 \%$. Naively, because a large $p_{\mathrm{T}}^{\text {cut }}$ reduces the background much more than the signal, a very hard $p_{\mathrm{T}}^{\text {cut }}$ may give the best significance. However, because the backgrounds are estimated from data, if the background rate is much smaller than that in the present data, the fractional uncertainty may increase. Thus we limit our chosen cuts to where $B$ is roughly in the same range as the current ATLAS backgrounds, where we assume that the uncertainty can be approximated by $\beta_{\text {tot }}=.25$ [43]. Nevertheless, an even harder cut might ultimately be effective.

Thus, the LHC14 has the potential to probe the lowmass region of the parameter space for an $\mathrm{SU}(2)$ doublet. Optimistically, if $P_{\text {mis }}=1$, we estimate a $5 \sigma$ discovery reach of about $\mu_{X}=150 \mathrm{GeV}$ and a $2 \sigma$ exclusion reach of about $\mu_{X}=170 \mathrm{GeV}$; however, a signal can still be found with larger $P_{\text {mis }}$. Further, some parameter space

\begin{tabular}{l|l|llll} 
& & \multicolumn{4}{|c}{$S\left(\mu_{X}\right.$ in $\left.(\mathrm{GeV})\right)$} \\
$p_{\mathrm{T}}\left(j_{1}\right)$ cut & $B / P_{\text {mis }}$ & 100 & 130 & 150 & 170 \\
\hline $200 \mathrm{GeV}$ & 227 & 1190 & 255 & 97 & 36 \\
$300 \mathrm{GeV}$ & 44.1 & 963 & 200 & 75 & 28 \\
$500 \mathrm{GeV}$ & 3.96 & 646 & 137 & 49 & 18
\end{tabular}

TABLE II. Number of the disappearing track background and signal events for $\sqrt{s}=14 \mathrm{TeV}, \mathcal{L}=3000 \mathrm{fb}^{-1}$, and $500 \mathrm{GeV}<$ $p_{\mathrm{T}}^{\text {track }}<1500 \mathrm{GeV}$.

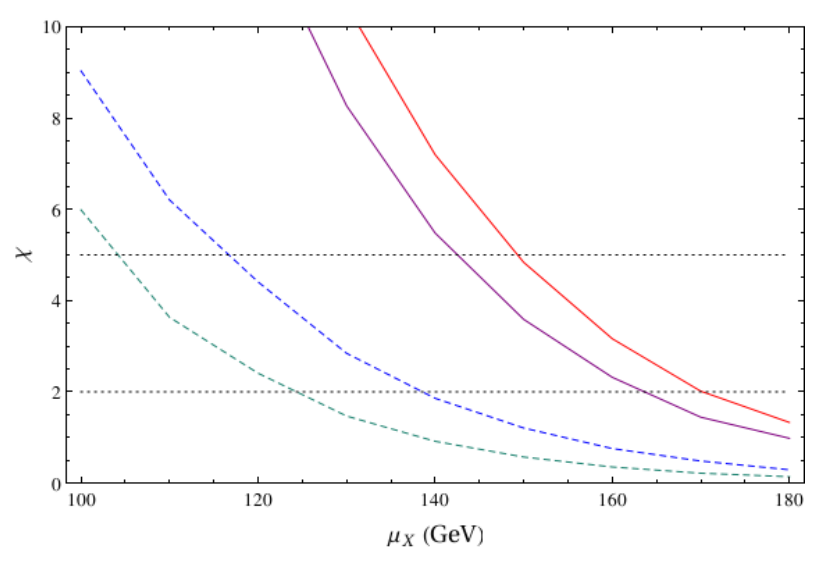

FIG. 4. The significance $\chi$ as a function of $\mu_{X}$ with $\sqrt{s}=$ $14 \mathrm{TeV}, 500 \mathrm{GeV}<p_{\mathrm{T}}^{\text {track }}<1500 \mathrm{GeV}$, and $\sigma_{B}=\beta_{\text {tot }} B$, $\beta_{\text {tot }}=.25$. The dashed curves are for $\mathcal{L}=300 \mathrm{fb}^{-1}$ assuming $P_{\text {mis }}=10$ and $p_{\mathrm{T}}\left(j_{1}\right)>300 \mathrm{GeV}$ (green, lower) or $P_{\text {mis }}=1$ and $p_{\mathrm{T}}\left(j_{1}\right)>200 \mathrm{GeV}$ (blue, upper). The solid curves are for $\mathcal{L}=3000 \mathrm{fb}^{-1}$ assuming $P_{\text {mis }}=10$ and $p_{\mathrm{T}}\left(j_{1}\right)>500$ $\mathrm{GeV}$ (purple, lower) or $P_{\mathrm{mis}}=1$ and $p_{\mathrm{T}}\left(j_{1}\right)>300 \mathrm{GeV}$ (red, upper). All $p_{T}\left(j_{1}\right)$ cuts have been chosen to optimize the significance as described in the text. Dotted lines indicate $2 \sigma$ exclusion and $5 \sigma$ discovery thresholds.

will likely be accessible at lower luminosities. However, the exact reach will depend on how the backgrounds and their uncertainties scale with energy, instantaneous luminosity, and $p_{\mathrm{T}}^{\text {cut }}$.

If a signal is detected, the track length and $p_{\mathrm{T}}^{\text {track }}$ distributions could provide clues about the type of particle that is detected. For example, because of the shortness of the $X^{ \pm}$lifetime, this model will have tracks with higher $p_{\mathrm{T}}$ and shorter path lengths, while other models with longer lifetimes that have so far avoided detection will have smaller production cross sections that compensate for the greater probability for long path lengths. 


\section{CONCLUSION}

We have explored the possibility that new vectorlike doublets may be present at the $\mathrm{TeV}$ scale. If stable, these particles must make up a tiny fraction of the dark matter. Nevertheless, they may be phenomenologically relevant. They could actually be the first signal observed at direct detection experiments, perhaps presenting a background to the true dark matter.

Comparing the two collider channels presented, the disappearing track channel has the potential to probe a significantly larger mass range than the monojet channel. Interestingly, it is this low mass window where the non-thermal cosmology realizes this scenario most easily, i.e. with the largest values of $c_{X}$, see Fig. 2. Observing larger-mass doublets at hadron colliders is challengingunless, perhaps, they are part of a larger dark sector that boosts either the production and/or the visibility of the events containing the $X$ particles. If a missing energy signal is found in the monojet channel, it will be difficult to determine what type of particle is responsible for it, and indeed whether it corresponds to a significant fraction of the dark matter. A much smaller set of models will (simultaneously) produce a disappearing track signature. Moreover, with enough statistics, further inferences can in principle be made from the lifetime distribution in the detector.

These added clues, perhaps along with material dependence at direct detection experiments, would be enough to indicate that a putative direct detection signal actually came from the "tip of the dark matter iceberg". A future lepton collider could also probe this scenario contingent on kinematic accessibility.

\section{ACKNOWLEDGMENTS}

The authors would like to thank Lisa Everett, Jack Kearney, Sven Krippendorf, Scott Watson, and Daniel Whiteson. The work of JH is supported by the National Science Foundation under Grant No. PHYS11-25915, and AP further acknowledges the KITP for support during his visit. The work of NO is supported by CAREER grant NSF-PHY 0743315. The work of AP is supported by DOE grant DE-SC0007859.
[1] D. Akerib et al. (LUX Collaboration), (2013), arXiv:1310.8214 [astro-ph.CO].

[2] E. Aprile et al. (XENON100 Collaboration), Phys.Rev.Lett. 109, 181301 (2012), arXiv:1207.5988 [astro-ph.CO].

[3] T. Cohen, J. Kearney, A. Pierce, and D. Tucker-Smith, Phys.Rev. D85, 075003 (2012), arXiv:1109.2604 [hepph].

[4] C. Cheung, L. J. Hall, D. Pinner, and J. T. Ruderman, JHEP 1305, 100 (2013), arXiv:1211.4873 [hep-ph].

[5] G. Duda, G. Gelmini, and P. Gondolo, Phys.Lett. B529, 187 (2002), arXiv:hep-ph/0102200 [hep-ph].

[6] G. Giudice and A. Masiero, Phys.Lett. B206, 480 (1988).

[7] H. P. Nilles, M. Srednicki, and D. Wyler, Phys.Lett. B120, 346 (1983); J. Frere, D. Jones, and S. Raby, Nucl.Phys. B222, 11 (1983); J. Derendinger and C. A. Savoy, ibid. B237, 307 (1984).

[8] R. Blumenhagen, M. Cvetič, and T. Weigand, Nucl. Phys. B771, 113 (2007), arXiv:hep-th/0609191; L. Ibanez and A. Uranga, JHEP 0703, 052 (2007), arXiv:hep-th/0609213 [hep-th]; B. Florea, S. Kachru, J. McGreevy, and N. Saulina, ibid. 0705, 024 (2007), arXiv:hep-th/0610003 [hep-th].

[9] M. W. Goodman and E. Witten, Phys.Rev. D31, 3059 (1985).
[10] R. Essig, Phys.Rev. D78, 015004 (2008), arXiv:0710.1668 [hep-ph].

[11] J. Halverson, Phys.Rev.Lett. 111, 261601 (2013), arXiv:1310.1091 [hep-th].

[12] M. Cvetič, J. Halverson, and P. Langacker, JHEP 11, 058 (2011), arXiv:1108.5187 [hep-ph]; M. Cvetič, J. Halverson, and H. Piragua, ibid. 1302, 005 (2013), arXiv:1210.5245 [hep-ph].

[13] N. Arkani-Hamed, S. Dimopoulos, and S. Kachru, (2005), arXiv:hep-th/0501082 [hep-th].

[14] R. Mahbubani and L. Senatore, Phys.Rev. D73, 043510 (2006), arXiv:hep-ph/0510064 [hep-ph].

[15] G. Belanger, F. Boudjema, A. Pukhov, and A. Semenov, (2013), arXiv:1305.0237 [hep-ph].

[16] N. Arkani-Hamed, A. Delgado, and G. Giudice, Nucl.Phys. B741, 108 (2006), arXiv:hep-ph/0601041 [hep-ph].

[17] P. Ade et al. (Planck Collaboration), (2013), arXiv:1303.5076 [astro-ph.CO].

[18] M. Dine and A. Kusenko, Rev.Mod.Phys. 76, 1 (2003), arXiv:hep-ph/0303065 [hep-ph].

[19] J. Beringer et al. (Particle Data Group), Phys.Rev. D86, 010001 (2012).

[20] P. Cushman, C. Galbiati, D. McKinsey, H. Robertson, T. Tait, et al., (2013), arXiv:1310.8327 [hep-ex]. 
[21] A. M. Green, PoS IDM2008, 108 (2008), arXiv:0809.1904 [astro-ph].

[22] H. Baer, A. Lessa, and W. Sreethawong, JCAP 1201, 036 (2012), arXiv:1110.2491 [hep-ph].

[23] G. B. Gelmini and P. Gondolo, Phys.Rev. D74, 023510 (2006), arXiv:hep-ph/0602230 [hep-ph]; G. Gelmini, P. Gondolo, A. Soldatenko, and C. E. Yaguna, ibid. D74, 083514 (2006), arXiv:hep-ph/0605016 [hep-ph].

[24] J. McDonald, Phys.Rev. D43, 1063 (1991).

[25] E. Aprile (XENON1T collaboration), (2012), arXiv:1206.6288 [astro-ph.IM].

[26] Similar statements can be made about operators arising from non-renormalizable Kahler potential terms e.g. $\phi^{\dagger} X \bar{X}$ after using the equation of motion for $X$.

[27] A. Heister et al. (ALEPH Collaboration), Phys.Lett. B533, 223 (2002), arXiv:hep-ex/0203020 [hep-ex]; G. Abbiendi et al. (OPAL Collaboration), Eur.Phys.J. C29, 479 (2003), arXiv:hep-ex/0210043 [hep-ex]; J. Abdallah et al. (DELPHI Collaboration), ibid. C34, 145 (2004), arXiv:hep-ex/0403047 [hep-ex]; M. Acciarri et al. (L3 Collaboration), Phys.Lett. B482, 31 (2000), arXiv:hep-ex/0002043 [hep-ex].

[28] Ref. [44] studies prospects for the vector boson fusion (VBF) channel but concludes that it is not sensitive to a pure Higgsino.

[29] S. D. Thomas and J. D. Wells, Phys.Rev.Lett. 81, 34 (1998), arXiv:hep-ph/9804359 [hep-ph].

[30] ATLAS Collaboration, ATLAS-CONF-2012-147, ATLAS-COM-CONF-2012-190 (2012).

[31] CMS Collaboration, CMS-PAS-EXO-12-048 (2013).

[32] J. Alwall, M. Herquet, F. Maltoni, O. Mattelaer, and T. Stelzer, JHEP 1106, 128 (2011), arXiv:1106.0522 [hep-ph].
[33] T. Sjostrand, S. Mrenna, and P. Z. Skands, JHEP 0605, 026 (2006), arXiv:hep-ph/0603175 [hep-ph].

[34] M. L. Mangano, M. Moretti, F. Piccinini, and M. Treccani, JHEP 0701, 013 (2007), arXiv:hep-ph/0611129 [hep-ph].

[35] S. Mrenna and P. Richardson, JHEP 0405, 040 (2004), arXiv:hep-ph/0312274 [hep-ph].

[36] J. Conway et al., "PGS - Pretty Good Simulation," (2009), http://physics.ucdavis.edu/ conway/ research/software/pgs/pgs4-general.htm.

[37] Another approach is to estimate the use of data-driven uncertainties to reduce the background uncertainty as in [45]. We found this approach tended to underestimate the uncertainties when applied to current experimental data $[30,31]$, and does not substantively change the conclusions.

[38] H. Baer, A. Mustafayev, and X. Tata, (2014), arXiv:1401.1162 [hep-ph].

[39] C. Han, A. Kobakhidze, N. Liu, A. Saavedra, L. Wu, et al., (2013), arXiv:1310.4274 [hep-ph].

[40] P. Schwaller and J. Zurita, (2013), arXiv:1312.7350 [hep$\mathrm{ph}]$.

[41] N. Zhou, D. Berge, L. Wang, D. Whiteson, and T. Tait, (2013), arXiv:1307.5327v2 (forthcoming) [hep-ex].

[42] G. Aad et al. (ATLAS Collaboration), Phys.Rev. D88, 112006 (2013), arXiv:1310.3675 [hep-ex].

[43] We also entertained the possibility that data-driven methods could decrease $\beta_{\text {tot }}$ as low as, e.g., .05 with softer cuts and larger $B$, but find harder cuts still produce better significance.

[44] A. G. Delannoy, B. Dutta, A. Gurrola, W. Johns, T. Kamon, et al., (2013), arXiv:1308.0355 [hep-ph].

[45] M. Drees, M. Hanussek, and J. S. Kim, Phys.Rev. D86, 035024 (2012), arXiv:1201.5714 [hep-ph]. 